\title{
Simultaneous Phase Separation and Pattern Formation in Chiral Active Mixtures
}

\author{
Demian Levis ${ }^{1,2,3, \text { a) and Benno Liebchen }}{ }^{4}$ \\ ${ }^{1)}$ CECAM Centre Européen de Calcul Atomique et Moléculaire, École Polytechnique Fédérale de Lausanne, Batochime, \\ Avenue Forel 2, 1015 Lausanne, Switzerland \\ ${ }^{2)}$ Departament de Física de la Matèria Condensada, Universitat de Barcelona, Martí i Franquès 1, E08028 Barcelona, \\ Spain \\ 3) UBICS University of Barcelona Institute of Complex Systems, Martí i Franquès 1, E08028 Barcelona, \\ Spain \\ 4) Institut für Theoretische Physik II: Weiche Materie, Heinrich-Heine-Universität Düsseldorf, D-40225 Düsseldorf, \\ Germany
}

(Dated: 25 January 2019)

Chiral active particles, or self-propelled circle swimmers, from sperm cells to asymmetric Janus colloids, form a rich set of patterns, which are different from those seen in linear swimmers. Such patterns have mainly been explored for identical circle swimmers, while real-world circle swimmers, typically possess a frequency distribution. Here we show that even the simplest mixture of (velocity-aligning) circle swimmers with two different frequencies, hosts a complex world of superstructures: The most remarkable example comprises a microflock pattern, formed in one species, while the other species phase separates and forms a macrocluster, coexisting with a gas phase. Here, one species microphaseseparates and selects a characteristic length scale, whereas the other one macrophase separates and selects a density. A second notable example, here occurring in an isotropic system, are patterns comprising two different characteristic length scales, which are controllable via frequency and swimming speed of the individual particles.

\section{INTRODUCTION}

Chirality, the property of a structure to be distinguishable from (or not superimposable with) its mirror image, plays an important role in all natural sciences. In physics, for example, the concept of chirality plays an important role from subatomic scales - for nucleonic mass generation - to astronomical scales - for the formation of galaxies, commonly showing a disk-like geometry with bright spiral arms witnessing ongoing star-formation. In biology, chirality shows up, for instance, in the double-helical structure of DNA, the shape of bacterial flagella or the anatomy of flatfish like Halibut. Interestingly, in many cases left- and right-handed chiral structures are not equally distributed: For instance, bacterial flagella and 19 out of 20 amino acids are left-handed, evoking questions regarding the origin and possible purpose of the prevalence of a certain handedness upon chiral structures. (Did it emerge before or from life, on earth, or does it have an extraterrestrial origin?)

Chirality also occurs in active matter, comprising selfpropelled particles such as microswimmers. Here, microswimmers with mirror-symmetric body parts swim linearly, whereas those with chiral body shapes (or body parts), show noisy circular trajectories in 2D and helical trajectories in $3 \mathrm{D}^{12}$. Biological examples of chiral microswimmers include sperm cells $3+5$ and $E$. Coli bacteria close to walls or interfaces 6 , both featuring chiral body parts determining the handedness of their swimming trajectories. Therefore, ensembles of chiral biological microswimmers often share the same chirality (monochirality), but show a distribution

\footnotetext{
${ }^{a)}$ Electronic mail: demian.levis@epfl.ch
}

of rotation frequencies. Conversely, man-made synthetic microswimmers, like asymmetric Janus-colloids 910 or granular microflyers 11 allow to engineer the handedness of a microswimmer on demand (e.g. via $3 \mathrm{D}$ printing 12 ). Thus, also polychiral mixtures can be produced, which in principle could be reduced to monochiral mixtures using chiral segregation schemes 13.15 .

Besides affecting the trajectories of single active particles, in free space or in crowded environments 16 , chirality can also have a spectacular impact on the collective behavior of circle swimmers. Specifically, for the single frequency (monochromatic) case, it has been shown that circle swimmers with a tendency to align can self-organize into synchronized rotating doublets 17 and large clusters $18 \mid 19$, providing a potential microscopic basis for the rotating ring-clusters observed in selfpropelled membrane-bound FtsZ-proteins $\sqrt{18 \mid 20}$. This class of circle swimmers can also self-organize into a pattern of rotating microflocks with a well-defined length-scale which can be controlled by the swimming speed and rotation frequency of the individual microswimmers 1921 , resembling the patterns seen in ensembles of sperm cells 3 . Circle swimmers with spherical body shapes, which do not align, but sterically repel each other can form hyperuniform states 22 and aggregate in (macro)clusters ${ }^{23 \mid 24}$ which can even counterrotate with respect to the surrounding gas 23 .

As compared to the monochromatic case, less is known about the patterns emerging in (aligning) circle swimmers with different frequencies. For such mixtures, broadly occurring both in nature and in the world of synthetic microswimmers, previous work has focused on synchronization of circle swimmers 15 , reporting the formation of counterrotating macroclusters as a side result and similar structures discussed in 25 . Here, we explore and characterize pattern formation in chiral active mixtures more systematically and provide a state 
diagram significantly extending the one in 15 . As our key result, we find that this phase diagram comprises a class of unexpected superstructures, occurring generically in a wide domain of parameter space. The most interesting example for such a superstructure emerges for a mixture of two species with significantly different intrinsic frequencies: they selforganize into a microflock pattern, formed in one species, coexisting with a macrocluster formed by the other species and a gas phase. This remarkable pattern unites microphase- and macrophase-separation: one species selects a length scale, the other one a density, both being characteristic, i.e. independent of system size. (Notice that these superstructures do not emerge from superimposed patterns formed by each species individually, since each of the species on its own would stay in the disordered phase.) When both species rotate sufficiently fast, they form a second type of superstructure given by a pattern comprising two characteristic length scales: to form this pattern, circle-swimmers also self-sort by chirality and form individual microflocks with a species-selective size. In each case, the length scales involved in the superstructures we report can be controlled by the properties of the individual components of the system (swimming speed and frequency), rather than requiring a more involved design of their interactions, as often required to control pattern formation. Therefore, mixtures of circle swimmers provide a route to the formation of controllable superstructures, which might serve as a useful design principle to create active materials.

\section{CHIRAL ACTIVE PARTICLE MODEL}

We consider $N$ overdamped circle-swimmers, at positions $\boldsymbol{r}_{i}(t)$, at time $t$, which self-propel with a constant speed $v_{0}$ in directions $\boldsymbol{n}_{i}(t)=\left(\cos \theta_{i}(t), \sin \theta_{i}(t)\right)$ in a square box of linear size $L$ with periodic boundary conditions. The orientation of particle $i$ changes due to an intrinsic frequency $\omega_{i}$, rotational diffusion and alignment interactions (of strength coefficient $K$ ) with its neighbors, yielding ${ }^{15 \mid 19}$

$$
\begin{aligned}
& \dot{\boldsymbol{r}}_{i}(t)=v_{0} \boldsymbol{n}_{i}(t) \\
& \dot{\theta}_{i}(t)=\omega_{i}+\frac{K}{\pi R^{2}} \sum_{j \in \partial_{i}} \sin \left(\theta_{j}(t)-\theta_{i}(t)\right)+\sqrt{2 D_{r}} \eta_{i}(t)
\end{aligned}
$$

The sum runs over all the neighbors $j$ at a distance less than the interaction radius $R$ to the $i$-th particle, $\eta$ represents a Gaussian white noise of zero mean and unit variance and $D_{r}$ is the rotational diffusion coefficient.

State of the art and limiting cases: Before discussing pattern formation in mixtures of circle swimmers, let us briefly review what is known for some relevant limiting cases of this model:

- (i) Individual circle swimmers: In the absence of interactions $(K=0)$, each circle-swimmer shows circular Brownian motion with an average radius $v_{0} / \omega_{l}^{26277}$.

- (ii) Linear Swimmers: When switching on aligning interactions $(K>0)$ but considering non-rotating swimmers $\omega_{i}=0$, particles tend to locally align their swimming direction 15 . This kind of ferromagnetic, or polar, coupling can lead to flocking 28 , which occurs when the coupling exceeds a critical strength $K>K_{c}$ and allows a macroscopic fraction of the system to balistically move in a preferred direction. This yields long-range order in a 2D system with local coupling 29 .

- (iii) Single frequency (monochromatic) circle swimmers: In the presence of rotations, the critical coupling strength $K_{c}$ does not change. However, despite having little impact on the transition to flocking itself, rotations (or active torques) dramatically change the collective behavior of polar active particles in the ordered phase. For slow rotations, circle-swimmers form a macroscopic rotating cluster which coarsens and scales with the system size at late times, whereas faster rotations lead to a pattern of synchronized rotating clusters, or microflocks, with a characteristic size. This size scales linearly with the single particle radius $\frac{1921}{}$ offering a way to control the assembly of chiral active particles.

- (iv) No self-propulsion: For several frequencies, but in the absence of self-propulsion $\left(v_{0}=0\right)$, eq. 2 reduces to the (noisy) Kuramoto model of phase synchronization 30 , and if rotations are also absent, to the XY model of magnetism 31 , in a 2D geometric network. The two latter models have been largely studied, and it is known that they cannot sustain global (longrange) order in $2 \mathrm{D}^{32[33}$. However, remarkably, we now know that self-propulsion $\left(v_{0}>0\right)$ allows for global synchronization, which can occur e.g. in the form of a mutual flocking phase generalizing the Toner-Tu phase to circle swimmers 15 .

In the following, we focus on pattern formation in mixtures of circle swimmers, which has been far less explored than the above limiting cases. For simplicity, we consider two species with frequencies $\omega_{1,2}$ which can either have the same sign, representing a monochiral mixture, or different signs.

Units, parameters and simulations: We use the interaction range $R$ and the inverse of the rotational diffusion coefficient, $1 / D_{r}$, as length and time units, respectively. We define the following dimensionless parameters: (i) mean density per species, $\rho_{\alpha}=N_{\alpha} R^{2} / L^{2}$ and overall mean density $\rho_{0}=N R^{2} / L^{2}$, where $N_{\alpha}$ is the number of particles of species $\alpha$, with intrinsic frequency $\omega_{\alpha}$; (ii) the reduced frequencies $\Omega_{i}=\omega_{i} / D_{r}$ (iii) the coupling strength $g=K /\left(4 \pi R^{2} D_{r}\right)$ and (iv) the Péclet number Pe $=v_{0} /\left(R D_{r}\right)$ (which we fix at $\mathrm{Pe}=2)$. For simplicity, we focus on the case of equal density per species $\rho_{1}=\rho_{2}=\rho_{0} / 2\left(\rho_{1}=\rho_{2}=\rho_{3}=\rho_{0} / 3\right.$ in section VII. To explore pattern formation in mixtures of circle swimmers, we use Brownian Dynamics simulations of $N=10^{3}$ up to $N=32 \times 10^{3}$ particles using an Euler integration scheme with a time step $\Delta t=10^{-3}$. We then analyze the system at long-times, after letting it relax for more than $10^{4}$ times the rotational diffusion time $\left(t D_{r}=10^{4}\right)$ from a random initial configuration. As shown in $\frac{15}{15}$, at the level of a coarse-grained description of the model eq. 1, 2, the disordered state generically looses stability at $g \rho_{0}=2$ (which is robust against ex- 


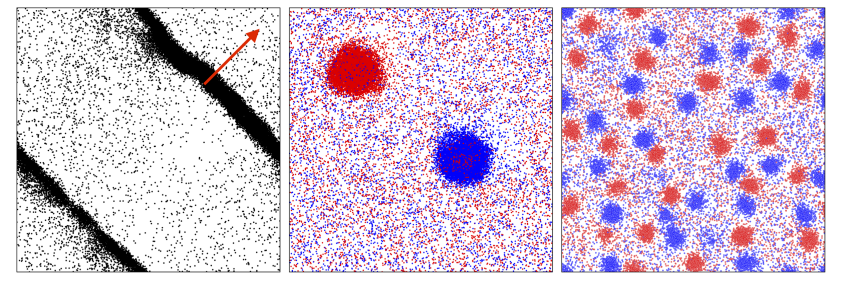

$\Omega$

FIG. 1. Snapshots based on Brownian Dynamics simulations for symmetric mixtures with $\Omega \equiv \Omega_{1}=-\Omega_{2}$ for increasing rotation frequency: (from left to right) $\Omega=0, \Omega=1$ and $\Omega=5$, at fixed $\rho_{0}=20$ and $N=32 \times 10^{3}$. The red arrow in the leftmost snapshot represents the average polarization of the particles in the dense band. Particles rotating at $\Omega$ are represented in blue and the ones rotating at $-\Omega$ in red. Linear swimmers are represented in black.

cluded volume interactions 21134 ). Thus, to study pattern formation, we choose $g \rho_{0}=3$ in the following. Note here, that in this case, the density per species is too low to induce pattern formation if it was the only species present; hence, particles of different species have to cooperate to form patterns.

\section{SYMMETRIC MIXTURES}

We first discuss unbiased symmetric mixtures $\Omega_{1}=-\Omega_{2}=$ $\Omega$. When the coupling is weak $\left(g \rho_{0} \lesssim 2\right)$ the positions and orientations of the particles are randomly distributed, leading to a disordered homogeneous gas-like phase (not shown). If $g \rho_{0}>2$, the disordered phase looses stability and a new state emerges, which may either be a uniform ordered phase, or a pattern.

Uniform ordered phase: If $g \rho_{0} \gg 2$ the system can settle in an ordered uniform phase which has been mainly explored for $\Omega=0$ (linear swimmers) where it features long-range polar order ${ }^{29}$ and giant density fluctuations ${ }^{35}$. Remarkably, a similar phase, the mutual flocking phase, occurs also for chiral particles of opposite handedness $(\Omega>0)$, which cooperate and mutually suppress their circular motion, forming two superimposed flocks at a relative angle to each other ${ }^{115}$.

Patterns: If $g \rho_{0} \gtrsim 2$ but not too large, the system forms patterns, comprising high density structures of polarly ordered particles which coexist with a disordered low density gas. We explore these patterns in the following: (i) For $\Omega=0$ (linear swimmers) the dense structures appear in the form of traveling bands $\sqrt{36}$, as shown in the leftmost snapshot Fig. 1 and discussed in the previous section. (ii) If $0 \ll \Omega \lesssim 1$ the onset of flocking is accompanied by the spatial segregation of particles by their chirality. Following segregation, chiral particles form polarly ordered rotating clusters which are roughly spherical (see Fig. 1), and coarsen as time proceeds. Notably, each cluster contains some particles of opposite chirality, which therefore rotate with a frequency opposite to their intrinsic one. The central panel of Fig. 11 shows such clusters at late times, which coexist with a disordered, low density background comprising particles of both species. Follow- ing their size and shape, we call them rotating macroclusters, or simply macrodrops. (iii) For $\Omega \gtrsim 1$ (e.g. $\Omega=5$, rightmost panel Fig. 1), circle swimmers self-sort by chirality, as in case (ii), but self-organize into a different state: a pattern of chiral rotating clusters, with a characteristic length-scale. The emergence of such a microflock pattern is associated with a short-wave length instability of the homogeneous flocking state, allowing to predict the size of the microflocks in the single-species case $\frac{19}{19}$. (For the present bichromatic case, a stability analysis of the mutual flocking phase would be needed to characterize the onset and length scales of microflock patterns, which is well-beyond the scope of the present article.)

Characterization of patterns: Macrodrops and Microflock Patterns To characterize the observed patterns, we analyze the size of the clusters in different regimes. We associate a characteristic length scale to the clusters based on the analysis of the pair correlation function

$$
N \rho_{0} G(r)=\left\langle\boldsymbol{\delta}\left(\left|\boldsymbol{r}-\boldsymbol{r}_{j}+\boldsymbol{r}_{i}\right|\right)\right\rangle
$$

and orientational self-correlation function

$$
C(r)=\left\langle\boldsymbol{n}_{i} \cdot \boldsymbol{n}_{j}\right\rangle_{r}
$$

where $\langle.\rangle_{r}$ denotes an average over all the pairs of particles at distance $r$. We thus define two length scales for such rotating clusters, representing a density- and an orientationalcorrelation length: we define $l$ via the criterion $G(l)=1$; and $\xi$ via $C(\xi)=e^{-1}$. In Fig. 2 (a) we show both length scales in the slow-rotating, macrodrop regime $(\Omega=0.5)$, and in the fast-rotating, microflock one $(\Omega=5)$ as a function of $\rho_{0}$ at fixed $g \rho_{0}=3$ (above the onset of flocking) and $N=16.10^{3}$. In each case $l, \xi$ are very similar to each other. Here, for $\Omega=5$ (microflock patterns) both length scales are independent of $\rho_{0}$ when keeping $g \rho_{0}$ constant. (These length scales have been recorded 'at late times' in the simulations, here, at $t D_{r}=25.10^{4}$; for a discussion about a possible coarsening of microflocks on timescales beyond those involved in the coarsening of the macrodroplets, $\sec ^{\frac{19}{19}}$ ) Conversely, for $\Omega=0.5$, the size of the macrodrop clearly decreases as the density increases, in a way which is consistent with a $l, \xi \sim 1 / \sqrt{\rho_{0}} \sim L$ scaling (at fixed particle number), as expected for systems undergoing phase separation. As further evidenced by the snapshots Fig. 2(b)-(e), the size of the macrodrops reduces when decreasing the system size (see (b), (d)), while it is the number of microflocks which increases when the density is reduced and not their size. In the macrodrop regime, for a given value of $g \rho_{0}, \mathrm{Pe}$ and $\Omega$, the system selects a density (the one of the macrodrops), while in the microflock regime the system selects a length scale.

\section{MONOCHIRAL MIXTURES}

We now consider mixtures of swimmers of the same chirality $\left(\Omega_{1} \times \Omega_{2}>0\right)$, which do not fully segregate. To see this, we first quantify 'segregation'. We compute the local density field of particles of species $1, \rho_{1}$, and species $2, \rho_{2}$. We then analyze the probability distribution $\mathscr{P}$ of their difference $\rho_{s}=\left(\rho_{1}-\rho_{2}\right) / \rho_{0}$. A region with an excess of particles 

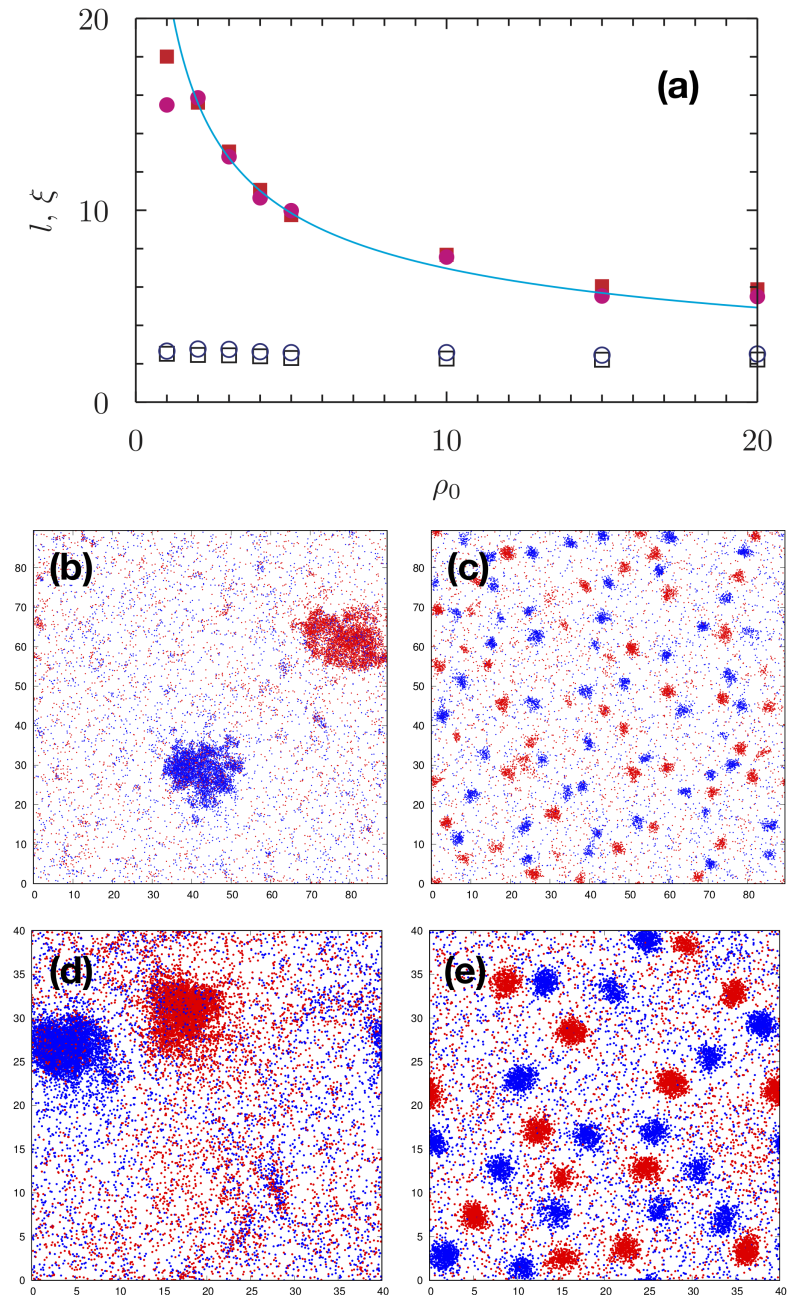

FIG. 2. (a) Characteristic length scales $l$ (rectangles) and $\xi$ (disks) as a function of $\rho_{0}$ at fixed $g \rho_{0}=3$ in the macrocluster $(\Omega=0.5$, in filled symbols) and microflock ( $\Omega=5$, in empty symbols) regime; the blue line shows a $1 / \sqrt{\rho_{0}}$ scaling law (at fixed particle number), i.e. $l, \xi \sim L$, showing that macroclusters scale linearly with the system size. Configuration snapshots of the system for $\rho_{0}=2, \Omega=0.5$ (b) and $\Omega=5$ (c) and $\rho_{0}=10, \Omega=0.5$ (d) and $\Omega=5$ (e). As the size of the system increases the size of the clusters in the macrocluster regime grows, while the number of microflocks in the system increases but keep approximately the same size. Here we used $N=16 \times 10^{3}$ particles.

of species 1 will be characterized by a peak- or shoulder of $\mathscr{P}$ at positive $\rho_{s}$, whereas peaks- or shoulders in $\mathscr{P}$ at negative $\rho_{s}$ stand for regions with an excess of particles of species 2. Thus, $\mathscr{P}$ allows us to quantify deviations from a homogeneous mixing of circle-swimmers. Representative examples of $\mathscr{P}\left[\rho_{s}\right]$ for several values of $\Omega_{1}$ at fixed $\Omega_{2}=1$ are shown in Fig. 3 (a). For the case $\Omega_{1}=\Omega_{2}$ (single species case), $\mathscr{P}\left[\rho_{s}\right]$ features a narrow Gaussian distribution around zero, stemming from particles in an incoherent gas-like state, and a broader Gaussian tail stemming from particles in a denser region (a macrodrop, not shown). As soon as $\Omega_{1}>\Omega_{2}$, the distributions become non-symmetric and develops a tail at values of $\rho_{s}>0$. Such a distribution signifies dense struc-

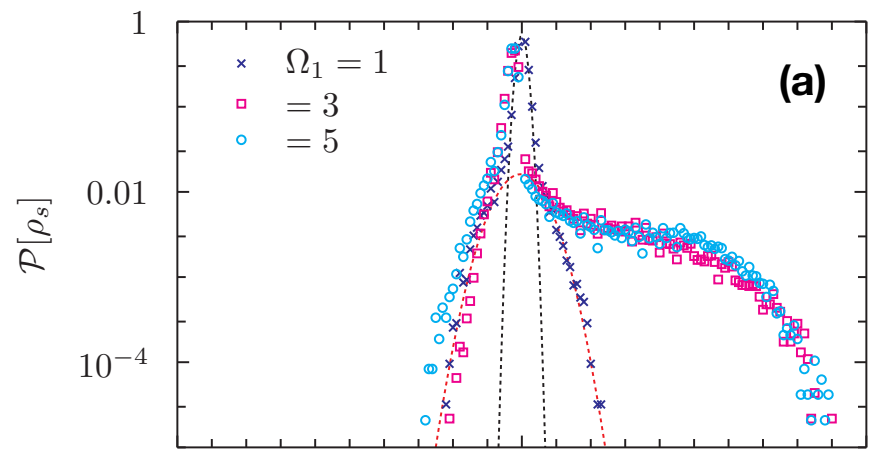

$-2.5 \quad 0$

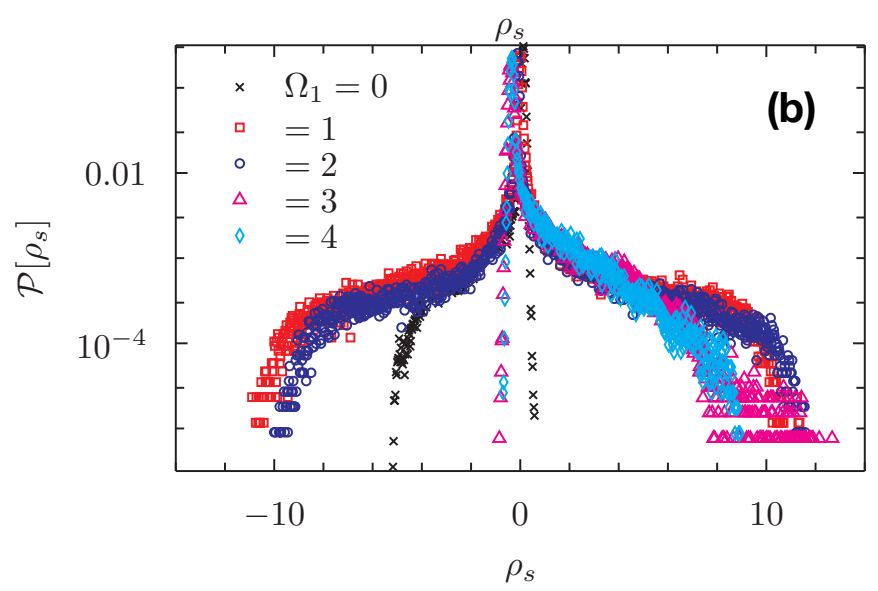

FIG. 3. Distribution of the local segregation factor $\mathscr{P}\left(\rho_{s}\right)$ with $\rho_{s}=\left(\rho_{1}-\rho_{2}\right) / \rho_{0}$ for several values of $\Omega_{1}$ (see key) for mono-chiral mixtures (fixed $\Omega_{2}=1$ ) (a) and for bi-chiral mixtures $\left(\Omega_{2}=-1\right.$ ) (b). The two dotted lines in the top panel correspond to two Gaussian distributions centered in zero with different variance.

tures with an excess of frequency- $\Omega_{1}$-particles, whereas the uniform background mainly contains $\Omega_{2}$-particles. (A configuration snapshot of such a state is shown in Fig. 5(d)). Thus, for monochiral mixtures, fast-rotating particles dominate the formation of the dense structures (i.e. of the pattern) while slower ones are partly relegated to the low density regions. (This is consistent with the fact that, at the level of field equation $\sqrt{19}$ for a single-species, the growth rate of the microflock-instability increases with the frequency.) For slower rotations, where the field equations for the singlespecies case do not show a (short-wavelength) microflock instability, but a long-wavelength instability 19 , this behaviour is less pronounced, and we observe a rotating macrocluster continaing a mixture of circle swimmers of both frequencies (Fig. 5b) - we refer to this case as mixing.

\section{SIMULTANEITY OF PATTERNS AND MACROCLUSTERS IN GENERIC MIXTURES}

Conversely to monochiral mixtures, where pattern formation is dominated by the faster rotating species, in symmetric mixtures both species of course behave equally when form- 
ing patterns. Here, we focus on circle swimmers with opposite handedness but different frequency $\left(\Omega_{1} \neq-\Omega_{2}\right)$ and show that they can generate remarkable patterns featuring two characteristic length scales.

To see this, let us first discuss the segregation behavior of non-symmetric bichiral mixtures (Fig. 3p). Conversely to the discussed mixtures where $\mathscr{P}\left(\rho_{s}\right)$ shows only one shoulder (Fig. 3a), remarkably, particles of opposite chirality can create two (asymmetric) shoulders in $\mathscr{P}\left(\rho_{s}\right)$ (Fig. 3 b). This leads to a rich set of possible patterns. For $\left|\Omega_{1}\right|<\left|\Omega_{2}\right|$ we find that particles of species 2 can self-organize into dense structures, while particles of species 1 remain rather uniformly distributed, as represented by the negative tail in the distribution for $\Omega_{1}=0$ Fig. 3 (b). For $\left|\Omega_{1}\right|=\left|\Omega_{2}\right|, \mathscr{P}\left[\rho_{s}\right]$ is symmetric with large tails at both $\rho_{s}>0$ and $\rho_{s}<0$, indicating the chiral sorting of particles into dense, counterrotating clusters of same density and size. For $\left|\Omega_{1}\right|>\left|\Omega_{2}\right|$ the distribution is nonsymmetric but features a broader tail at $\rho_{s}>0$, corresponding to a tendency of the system to generate dense structures made by a larger fraction of particles of species 1 . A configuration snapshot of the system in this case is shown in Fig. 5(e): highfrequency swimmers form microflocks while lower-frequency ones form a coexisting single macrocluster. This case represents an example of a state where one species phase separates (the size of the macrocluster scales with the system size) and the other species forms a pattern with a characteristic length scale. For a higher frequency dispersion [see snapshot Fig. 5 (f)], particles of species 1 quickly form microflocks, leaving no room for slower particles of species 2 to aggregate, as the peak of $\mathscr{P}\left[\rho_{s}\right]$ for $\Omega_{1}=3$ and $\Omega_{1}=4$ at small negative values of $\rho_{s}$ and the tail at large positive values of $\rho_{s}$ shows [see Fig. 3 (b)]. In this case we therefore observe a pattern occurring for one species, whereas the second species is in the uniform gas phase. Finally, for $\left|\Omega_{2}\right|>2$, microflock patterns can occur in both species, as the snapshot Fig. 3 (g) shows; here, remarkably, the resulting pattern comprises two different length scales. (Note here, that the observed patterns can not be viewed as a simple superposition of patterns formed by both species individually; instead, at $g \rho_{0}=3 / 2<2$, each of the species on its own would be in the disordered phase.)

To characterize these patterns, we generalize the definition used for symmetric mixtures to define length scales $l_{\alpha}, \xi_{\alpha}$ $(\alpha \in\{1,2\})$, associated with the density and orientation correlations for each species. We define $l_{\alpha}$ via $G_{\alpha}\left(l_{\alpha}\right)=1$, where $G(r)$ is the pair correlation function, which is in turn defined via $N \rho_{0} G_{\alpha}(r)=\left\langle\boldsymbol{\delta}\left(\left|\boldsymbol{r}-\boldsymbol{r}_{j}^{\alpha}+\boldsymbol{r}_{i}^{\alpha}\right|\right)\right\rangle$, where averages are performed over the particles, indexed with $i, j$. From the partial orientational correlation function $C_{\alpha}(r)$, defined via $C^{\alpha}(r)=\left\langle\boldsymbol{n}_{i}^{\alpha} \boldsymbol{n}_{j}^{\alpha}\right\rangle$, we define $\xi_{\alpha}$ via $C\left(\xi_{\alpha}\right)=1 / e$. In Fig. 4. we show these length scales as a function of $\Omega_{1}$ at fixed $\Omega_{2} \equiv-1$ (a) and $\Omega_{2}=-2$ (b). In both cases, as $\Omega_{1}$ increases, the characteristic length scale decreases, i.e. faster rotations induce smaller structures. For $\Omega_{2}=-1$ [see Fig. 4(a)], $l_{1}$ and $\xi_{1}$ decrease roughly linearly with $\Omega_{1}$, while the length scales associated to particles of species 2 saturate at some comparatively small values $\left(l_{2}=0\right.$ and $\left.\xi_{2} \approx 2\right)$ for $\Omega_{1} \geq 3$, as expected from the previous inspection of the distribution functions Fig 3 (b). In this regime, i.e. for large enough $\Omega_{1}$, particles of species
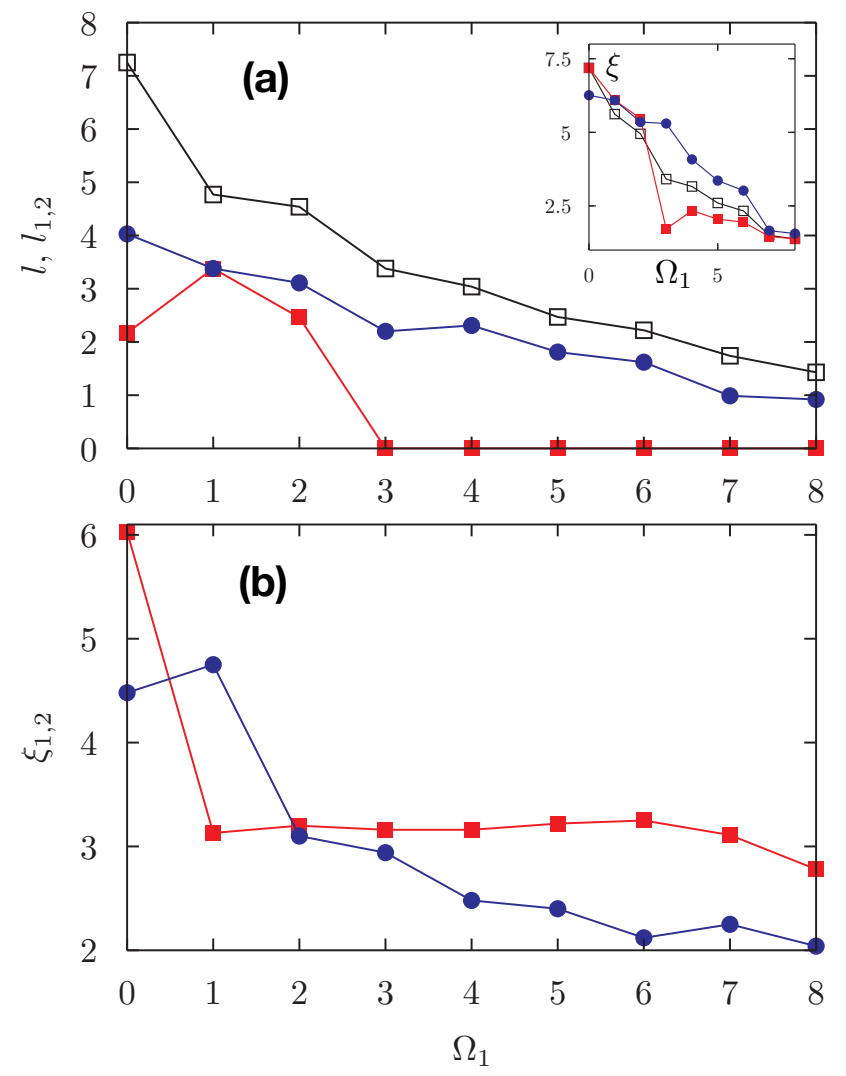

FIG. 4. (a): Length-scales associated to particle clustering, $l$ (in empty symbols), $l_{1}$ (in blue) and $l_{2}$ (in red), as a function of $\Omega_{1}$ at fixed $\Omega_{2}=-1$. The inset shows the correlation length $\xi$ (empty symbols), $\xi_{1}$ (in blue) and $\xi_{2}$ (in red). (b): $\xi_{1}$ (in blue) and $\xi_{2}$ (in red), as a function of $\Omega_{1}$ at fixed $\Omega_{2}=-2$.

2 are not able to form segregated dense structures, such that the pattern shows a single characteristic length scale. Interestingly, the situation changes for larger $\Omega_{2}$, providing a rich pattern formation scenario. Here, as shown in Fig. 4(b), two finite length scales associated to particles of species 1 and 2 can coexist. For $\Omega_{1} \geq 1$, particles of species 2 form clusters of a given size $\xi_{2} \approx 3$, while, by increasing $\Omega_{1}$, particles of species 1 form structures of smaller size. In this regime, the emerging patterns feature two characteristic length scales (see snapshot Fig. $5(\mathrm{~g}))$.

\section{PHASE DIAGRAM}

To provide an overview of the possible patterns seen in mixtures of circle swimmers, we now summarize our findings in the state diagram Fig. 5 Each symbol in the $\left(\Omega_{1}-\Omega_{2}\right)$-plane corresponds to a simulation. The pink area shows parameter regimes leading to microflock patterns while the blue one corresponds to the rotating macrodrop regime. Here, rectangular symbols represent states where swimmers of opposite chirality segregate into distinct dense structures, which can be either microflocks (giving rise to a two-length-scale pattern) or macrodrops. We distinguish five regimes, which we link to 

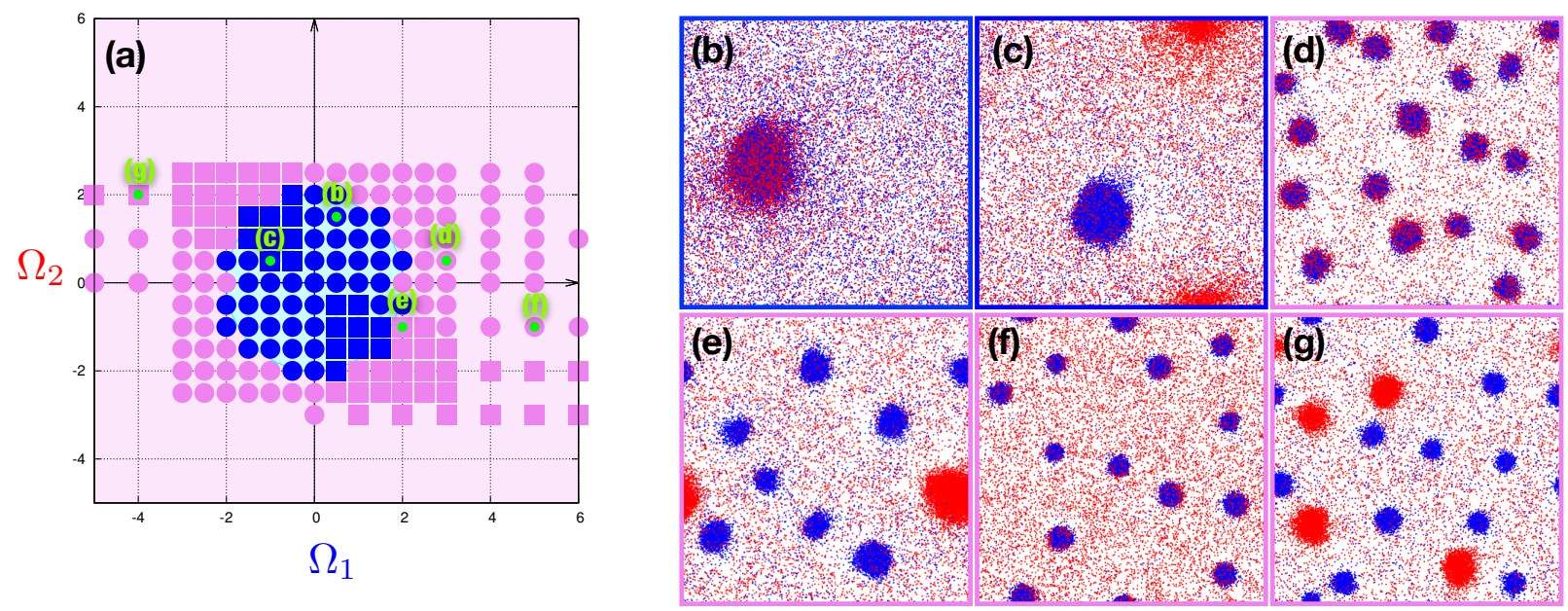

FIG. 5. State diagram of two-species mixtures of aligning circle-swimmers with rotation frequency $\Omega_{1}$ and $\Omega_{2}$ (a), together with representative snapshots at different points in the $\left(\Omega_{1}, \Omega_{2}\right)$ plane: (b): $(0.5,1.5) ;(\mathrm{c}):(-1,0.5) ;(\mathrm{d}):(3,0.5) ;(\mathrm{e}):(2,-1) ;(\mathrm{f}):(5 ;-1) ;(\mathrm{g}):(-4 ; 2)($ their location is indicated in the phase diagram (a) by green dots). Particles rotating at frequency $\Omega_{1}$ are represented in blue, while particles at frequency $\Omega_{2}$ are in red. Blue symbols in (a) denote macrocluster states, pink ones microflock states. Square symbols correspond to states where particles of opposite chirality segregate into different dense structures. The remaining symbols correspond to states where species segregation, if any, does not lead to the formation of species-specific clusters. Note that both macroclusters and microflock patterns of opposite chirality can appear, as shown in $(\mathrm{g})$ and $(\mathrm{c})$, respectively (and represented by blue and violet squares in the phase diagram).

the snapshots discussed earlier.

For $\Omega_{1}^{2}+\Omega_{2}^{2} \lesssim 2$, the system forms macroclusters. Here, we can either have (i) a single macrocluster containing a mixture of circle swimmers, which occurs in the monochiral case (see Fig. 5(b)) or by (ii) the formation of two macroclusters of opposite handedness (segregation), which generically feature different densities (see Fig. 5(c)). For $\Omega_{1}^{2}+\Omega_{2}^{2} \gtrsim 2$ microflock patterns emerge. Here, as discussed in the previous section, we can distinguish three different regimes, characterized by: (iii) the formation of microflocks made of a larger fraction of fast-rotating particles (see Fig. 5(d) and movie 1 in the $\mathrm{SM}^{37}$ for the mono-chiral case, and Fig. 5 (f) and movie $3^{37}$ for the bi-chiral one); (iv) the simultaneous formation of microflocks and macrodrops composed of swimmers of opposite chirality (see Fig. 5 (e) and movie 237); (v) the formation of microflocks of different size and handedness (see Fig. 5 (g)). The pattern in regimes (iii) and (iv) is characterized by a single length-scale while, strikingly, a two-length-scale pattern emerges in regime (v).

\section{MULTI-SPECIES MIXTURES}

Finally, we briefly comment on generalizations (i) to three species rotating at frequencies $\Omega_{1}, \Omega_{2}$ and $\Omega_{3}$ with $\rho_{1}=\rho_{2}=$ $\rho_{3}$ and (ii) to a continuous distribution of frequencies where each swimmers' frequency $\Omega_{i}$ is picked from a uniform distribution $U\left(\Omega_{a} ; \Omega_{b}\right)$.

We show in Fig. 6 configuration snapshots for four cases, using both mono-chiral and bi-chiral mixtures. As expected from our discussion above, fast-rotating particles lead to the formation of dense structures, while slowly-rotating ones largely remain in the disordered background. The size of the
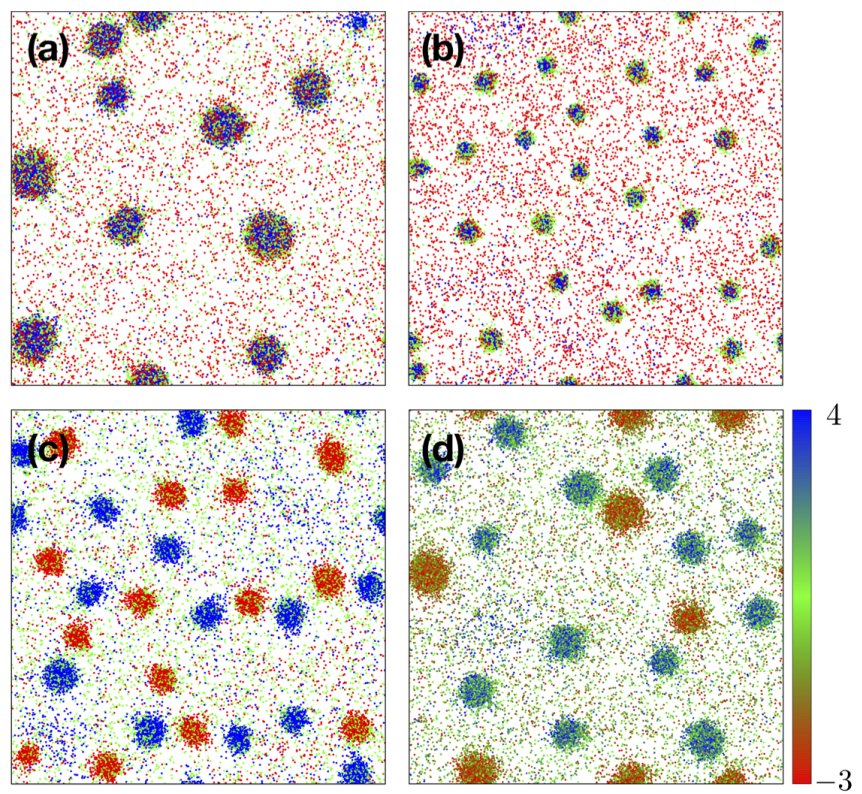

FIG. 6. Late-time snapshots for three-species mixtures with frequency $\left(\Omega_{1}, \Omega_{2}, \Omega_{3}\right)=(0,2,4)$ (a), $(-2,4,8)$ (b) and $(-3,0,3)$ (c) and for a continuous mixture of swimmers with uniformly distributed intrinsic frequencies with $\Omega_{a}=-3$ and $\Omega_{b}=4$ (d). Particles of each species are colored according to their frequency: $\Omega_{1}$-particles are in red, $\Omega_{2}$ in green and $\Omega_{3}$ in blue and using a the color code shown in (d) in the continuous polychromatic case.

microflock patterns is smaller for larger frequencies (see Fig. 6 (b)). For a large enough frequency difference between particles of opposite chirality, microflocks of opposite chirality emerge (see Fig. 6(c-d)), giving rise to, eventually, a pattern 
characterized by two different length scales [Fig. 6(d)].

\section{CONCLUSIONS}

We have shown that mixtures of circle swimmers with a tendency to align, show a spectrum of unusual structure formation scenarios. While bichiral mixtures tend to spatially segregate and hence self-sort by their chirality, circle swimmers of the same chirality can cooperatively form dense clusters, i.e. they mix. Within both regimes, segregation and mixing, chiral active particles can either form macroclusters (or macrodrops) with a size scaling with the system size or microflock patterns with a characteristic self-limited size. One particularly interesting scenario occurs when one species rotates much faster and opposite to the other one: here, a phase-separating macrocluster and a microflock pattern can simultaneously exist, albeit none of the two species would form structures on its own. Finally, also the case where both species rotate sufficiently fast in opposite directions is remarkable: here, they form a pattern of clusters comprising two length scales which can be individually controlled by the frequency or self-propulsion velocity of the particles of each species.

${ }^{1}$ H. Löwen, "Chirality in microswimmer motion: From circle swimmers to active turbulence," Eur. Phys. J. Spec. Top. 225, 2319 (2016).

${ }^{2}$ B. Friedrich, "Hydrodynamic synchronization of flagellar oscillators," Eur. Phys. J. Spec. Top. 225, 2353 (2016).

${ }^{3}$ I. H. Riedel, K. Kruse, and J. Howard, "A self-organized vortex array of hydrodynamically entrained sperm cells," Science 309, 300 (2005).

${ }^{4}$ Y. Yang, J. Elgeti, and G. Gompper, "Cooperation of sperm in two dimensions: synchronization, attraction, and aggregation through hydrodynamic interactions," Phys. Rev. E 78, 061903 (2008).

${ }^{5}$ J. Elgeti, R. G. Winkler, and G. Gompper, "Physics of microswimmers single particle motion and collective behavior: a review," Rep. Prog. Phys. 78, 056601 (2015).

${ }^{6}$ K. Maeda, Y. Imae, J.-I. Shioi, and F. Oosawa, "Effect of temperature on motility and chemotaxis of escherichia coli." J. Bacteriol. 127, 1039-1046 (1976).

${ }^{7}$ W. R. DiLuzio, L. Turner, M. Mayer, P. Garstecki, D. B. Weibel, H. C. Berg, and G. M. Whitesides, "Escherichia coli swim on the right-hand side," Nature 435, 1271 (2005).

${ }^{8}$ E. Lauga, W. R. DiLuzio, G. M. Whitesides, and H. A. Stone, "Swimming in circles: motion of bacteria near solid boundaries," Biophys. J. 90, 400 (2006).

${ }^{9}$ F. Kümmel, B. ten Hagen, R. Wittkowski, I. Buttinoni, R. Eichhorn, G. Volpe, H. Löwen, and C. Bechinger, "Circular motion of asymmetric self-propelling particles," Phys. Rev. Lett. 110, 198302 (2013).

${ }^{10}$ M. S. D. Wykes, J. Palacci, T. Adachi, L. Ristroph, X. Zhong, M. D. Ward, J. Zhang, and M. J. Shelley, "Dynamic self-assembly of microscale rotors and swimmers," Soft Matter 12, 4584 (2016).

${ }^{11}$ C. Scholz, S. Jahanshahi, A. Ldov, and H. Löwen, "Inertial delay of selfpropelled particles," arXiv preprint arXiv:1807.04357 (2018).

${ }^{12}$ C. Scholz, S. D' Silva, and T. Pöschel, "Ratcheting and tumbling motion of vibrots," New J. Physics 18, 123001 (2016).
${ }^{13}$ M. Mijalkov and G. Volpe, "Sorting of chiral microswimmers," Soft Matter 9, 6376 (2013).

${ }^{14} \mathrm{Q}$. Chen and B.-q. Ai, "Sorting of chiral active particles driven by rotary obstacles," J. Chem. Phys. 143, 09B612_1 (2015).

${ }^{15}$ D. Levis, I. Pagonabarraga, and B. Liebchen, "Activity induced synchronization," arXiv preprint arXiv:1802.02371 (2018).

${ }^{16} \mathrm{O}$. Chepizhko and T. Franosch, "Ideal circle microswimmers in crowded media," Soft Matter, Accepted Manuscript (2018).

${ }^{17}$ A. Kaiser and H. Löwen, "Vortex arrays as emergent collective phenomena for circle swimmers,” Phys. Rev. E 87, 032712 (2013).

${ }^{18}$ J. Denk, L. Huber, E. Reithmann, and E. Frey, "Active curved polymers form vortex patterns on membranes," Phys. Rev. Lett. 116, 178301 (2016).

${ }^{19} \mathrm{~B}$. Liebchen and D. Levis, "Collective behavior of chiral active matter: pattern formation and enhanced flocking," Phys. Rev. Lett. 119, 058002 (2017).

${ }^{20} \mathrm{M}$. Loose and T. J. Mitchison, "The bacterial cell division proteins ftsa and ftsz self-organize into dynamic cytoskeletal patterns," Nat. Cell. Biol. 16, 38 (2014)

${ }^{21}$ D. Levis and B. Liebchen, "Micro-flock patterns and macro-clusters in chiral active brownian disks," J. Phys. Cond. Matter 30, 084001 (2018).

${ }^{22}$ Q.-1. Lei, M. P. Ciamarra, and R. Ni, "Non-equilibrium strong hyperuniform fluids of athermal active circle swimmers with giant local fluctuations," arXiv preprint arXiv:1802.03682 (2018).

${ }^{23}$ G.-J. Liao and S. H. Klapp, "Clustering and phase separation of circle swimmers dispersed in a monolayer," Soft Matter 14, 7873 (2018).

${ }^{24}$ D. Reichhardt and C. J. O. Reichhardt, "Reversibility, pattern formation and edge transport in active chiral and passive disk mixtures," arXiv preprint arXiv:1812.04150 (2018).

${ }^{25}$ B.-q. Ai, Z.-g. Shao, and W.-r. Zhong, "Mixing and demixing of binary mixtures of polar chiral active particles," Soft matter 14, 4388 (2018).

${ }^{26}$ S. van Teeffelen and H. Löwen, "Dynamics of a brownian circle swimmer," Phys. Rev. E 78, 020101 (2008).

${ }^{27}$ F. J. Sevilla, "Diffusion of active chiral particles," Phys. Rev. E 94, 062120 (2016).

${ }^{28}$ T. Vicsek and A. Zafeiris, “Collective motion,” Phys. Rep. 517, 71 (2012).

${ }^{29} \mathrm{~J}$. Toner and Y. Tu, "Long-range order in a two-dimensional dynamical xy model: how birds fly together," Phys. Rev. Lett. 75, 4326 (1995).

${ }^{30}$ J. A. Acebrón, L. L. Bonilla, C. J. Pérez-Vicente, F. Ritort, and R. Spigler, "The kuramoto model: A simple paradigm for synchronization phenomena," Rev. Mod. Phys. 77, 137 (2005).

${ }^{31} \mathrm{~V}$. Berezinskii, "Destruction of long-range order in one-dimensional and two-dimensional systems having a continuous symmetry group i. classical systems," Sov. Phys. JETP 32, 493-500 (1971).

${ }^{32} \mathrm{H}$. Daido, "Lower critical dimension for populations of oscillators with randomly distributed frequencies: a renormalization-group analysis," Phys. Rev. Lett. 61, 231 (1988).

${ }^{33}$ N. D. Mermin and H. Wagner, "Absence of ferromagnetism or antiferromagnetism in one-or two-dimensional isotropic heisenberg models," Phys. Rev. Lett. 17, 1133 (1966).

${ }^{34}$ A. Martín-Gómez, D. Levis, A. Díaz-Guilera, and I. Pagonabarraga, "Collective motion of active brownian particles with polar alignment," Soft Matter 14, 2610 (2018).

${ }^{35} \mathrm{~V}$. Narayan, S. Ramaswamy, and N. Menon, "Long-lived giant number fluctuations in a swarming granular nematic," Science 317, 105 (2007).

${ }^{36}$ H. Chaté, F. Ginelli, G. Grégoire, and F. Raynaud, "Collective motion of self-propelled particles interacting without cohesion,” Phys. Rev. E 77, 046113 (2008).

${ }^{37}$ See Supplementary Material at doi:....

${ }^{38}$ C. Hoell, H. Löwen, and A. M. Menzel, "Dynamical density functional theory for circle swimmers," New J. Phys. 19, 125004 (2017).

${ }^{39}$ F. D. C. Farrell, M. C. Marchetti, D. Marenduzzo, and J. Tailleur, "Pattern formation in self-propelled particles with density-dependent motility," Phys. Rev. Lett. 108, 248101 (2012). 